IUCrJ

ISSN 2052-2525

BIOLOGY|MEDICINE

Received 6 April 2021

Accepted 1 June 2021

Edited by J. L. Smith, University of Michigan, USA

Keywords: EptA; phosphoethanolamine transferase; small-angle $\mathrm{X}$-ray scattering; tryptophan fluorescence; conformational flexibility; enzyme substrate recognition.

Supporting information: this article has supporting information at www.iucrj.org

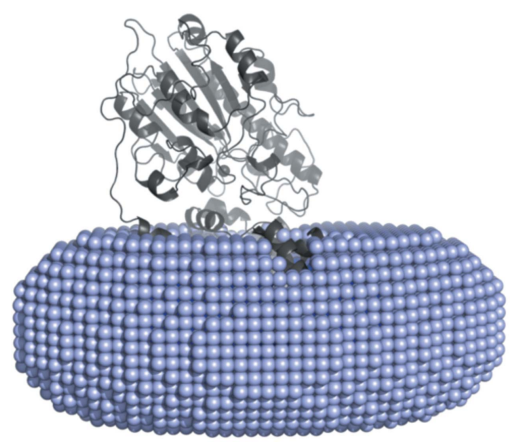

OPEN ๑ ACCESS

\section{Conformational flexibility of EptA driven by an interdomain helix provides insights for enzyme- substrate recognition}

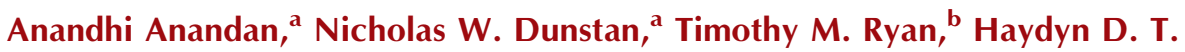 \\ Mertens, ${ }^{\mathrm{c}}$ Katherine Y. L. Lim, ${ }^{\mathrm{d}}$ Genevieve L. Evans, ${ }^{\mathrm{a}}$ Charlene M. Kahler ${ }^{\mathrm{d}}$ and Alice \\ Vrielink $^{\mathrm{a} *}$
}

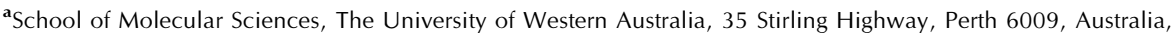
${ }^{\mathbf{b}}$ Australian Synchrotron, 800 Blackburn Road, Clayton, Victoria 3168, Australia, ${ }^{\mathbf{C}}$ European Molecular Biology Laboratory, Hamburg Unit, DESY, Notkestrasse 85, 22607 Hamburg, Germany, and ${ }^{\mathbf{d}}$ The Marshall Centre for Infectious Diseases Research and Training, School of Biomedical Sciences, University of Western Australia, 35 Stirling Highway, Perth, Western Australia 6009, Australia. *Correspondence e-mail: alice.vrielink@uwa.edu.au
}

Many pathogenic gram-negative bacteria have developed mechanisms to increase resistance to cationic antimicrobial peptides by modifying the lipid A moiety. One modification is the addition of phosphoethanolamine to lipid A by the enzyme phosphoethanolamine transferase (EptA). Previously we reported the structure of EptA from Neisseria, revealing a two-domain architecture consisting of a periplasmic facing soluble domain and a transmembrane domain, linked together by a bridging helix. Here, the conformational flexibility of EptA in different detergent environments is probed by solution scattering and intrinsic fluorescence-quenching studies. The solution scattering studies reveal the enzyme in a more compact state with the two domains positioned close together in an $n$-dodecyl- $\beta$-D-maltoside micelle environment and an open extended structure in an $n$-dodecyl-phosphocholine micelle environment. Intrinsic fluorescence quenching studies localize the domain movements to the bridging helix. These results provide important insights into substrate binding and the molecular mechanism of endotoxin modification by EptA.

\section{Introduction}

Pathogenic Gram-negative bacteria have evolved numerous mechanisms to evade the human immune system and developed widespread resistance to host-derived antimicrobials and traditional antibiotics. One such mechanism is to substitute the negatively charged phosphate lipid A head group with phosphoethanolamine (PEA) or 4-amino-arabinose residues. Lipid A phosphoethanolamine transferase (EptA) transfers PEA from phosphatidylethanolamine to the 1 and $4^{\prime}$ positions of lipid A [Fig. 1(a)] (Cox et al., 2003). This modification makes the bacterial surface less negatively charged (AnayaLópez et al., 2013; Peschel, 2002) and repels the binding of cationic antimicrobial peptides to the surface thus conferring resistance (John et al., 2012; Trombley et al., 2015). EptA is found in a number of clinically relevant Gram-negative bacteria (Huang et al., 2018) and, given its important biological role in reducing the innate immune response, this enzyme has been a target for the development of potential inhibitors as therapeutic agents to treat multi-drug resistant bacterial infections (Kahler et al., 2018). Towards this aim, and in order to better understand the molecular mechanism involved in substrate binding and enzyme catalysis, the 


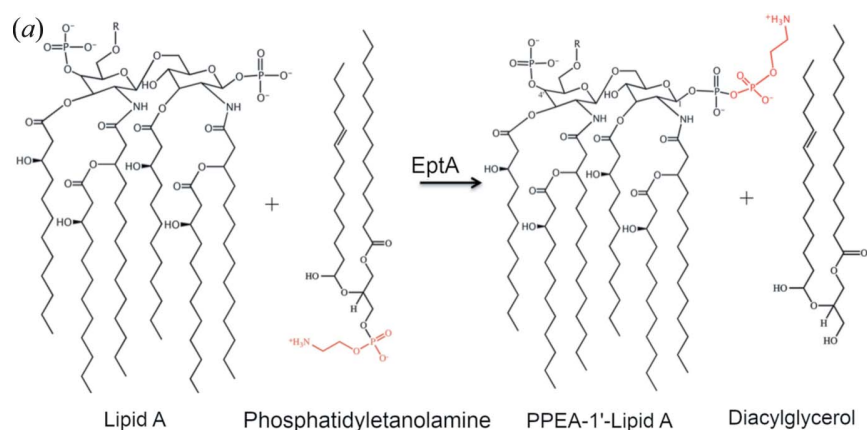

(b)

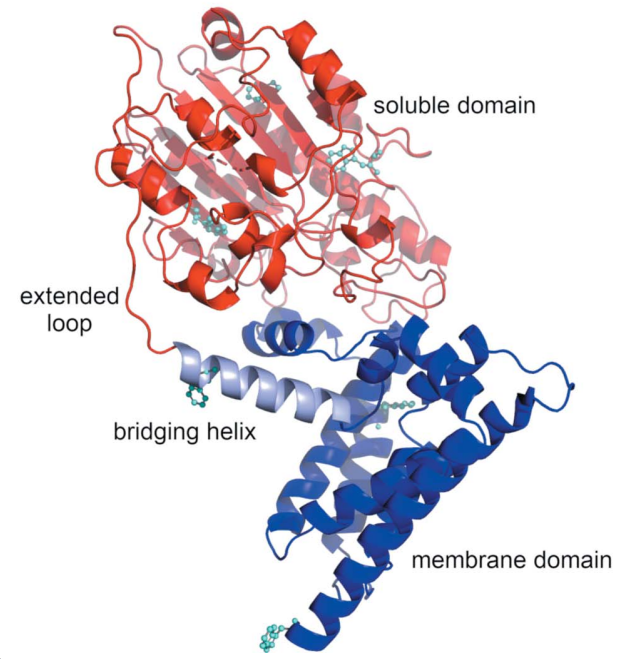

Figure 1

Catalytic reaction and structure of EptA. (a) Reaction catalysed by EptA. (b) Secondary structure representation of EptA from $N$. meningitidis (PDB entry 5 fgn). The $\mathrm{N}$-terminal transmembrane domain is represented in dark blue and the $\mathrm{C}$-terminal soluble domain is represented in red. The bridging helix that connects the transmembrane domain and soluble domain is represented in pale blue. The tryptophan residues in the protein are depicted as cyan balls and sticks.

structure of EptA was solved by X-ray crystallography (Anandan et al., 2017).

The crystal structure of EptA revealed a protein fold with a transmembrane domain and a periplasmic-facing soluble domain linked by an extensive loop and a bridging helix [Fig. 1(b)] (Anandan et al., 2017). The structure also revealed a tetrahedrally co-ordinated $\mathrm{Zn}^{2+}$ ion close to the catalytic nucleophile, Thr280, and a bound $n$-dodecyl- $\beta$-D-maltoside (DDM) molecule between the soluble and transmembrane domains near to the active site.

The ability of the enzyme to cleave PEA from phosphatidylethanolamine in the absence of lipid A (Anandan et al., 2017) along with the conformation observed in the crystal structure suggests that the two lipid substrates, varying in size, might sequentially access the active site invoking a ping-pong mechanism of catalysis (Wanty et al., 2013). The bound DDM molecule in the structure appears to position the soluble domain relatively close to the transmembrane domain, thereby locking the enzyme in a conformation that may mimic the phosphatidylethanolamine-bound state of EptA. In this conformation the binding site would be insufficient in size to accommodate a large lipid A substrate. This further suggests that the enzyme may adopt an alternate conformation to perform the transfer of phosphoethanolamine from the active site Thr280 to the lipid A headgroup. Towards establishing the ability of EptA to adopt different conformational poses, the enzyme was also solubilized and purified in $n$-dodecylphosphocholine (DPC) detergent micelles, differing from DDM in both structural and ionic properties. Such an approach has enabled studies of the conformational flexibility of other membrane proteins, such as PhoPQ-activated gene $\mathrm{P}$ (PagP) in lauroyl dimethylamine- $N$-oxide (LDAO) micelles and DPC micelles (Hwang et al., 2004).

DPC is a zwitterionic detergent containing a phosphocholine head group, highly prevalent among phospholipids of membranes, and a dodecyl alkyl tail. Although DPC has gained a reputation for being a harsh detergent, destabilizing and distorting protein structures (Chipot et al., 2018), it has also provided structural insights into flexible protein systems such as the phosphate transporter Pho89 (Sengottaiyan et al., 2013) and potassium channel KcsA (Imai et al., 2012). The effect of DPC on a protein is highly dependent on the specific structure and fold of the macromolecule. Hence, assessment of the protein structural integrity in DPC micelles is essential before undertaking any conformational investigation and interpretations of such studies.

EptA maintains its secondary and tertiary structural integrity in both DDM and DPC micelles as shown by circular dichroism and thermal shift assays, respectively (Anandan et al., 2017). Limited proteolysis and intrinsic fluorescence experiments performed with EptA in DDM micelles and in DPC micelles suggested that the enzyme adopts different conformations in different detergent micelles (Anandan et al., 2017). Furthermore, in silico molecular dynamics simulations of the enzyme in a bilayer containing dipalmitoyl-sn-glycero-3phosphoethanolamine lipids indicated that the soluble domain could adopt an alternate position relative to the transmembrane domain (Anandan et al., 2017). In this simulation the soluble domain was observed to 'roll over' the membrane surface yielding an elongated conformation distinct from that observed in the crystal structure. The molecular dynamics study provided impetus to study the conformational flexibility of the enzyme using different experimental biophysical methods.

Structural investigation of EptA in DPC micelles by crystallography has been challenging so far due to the lack of crystal formation. Small-angle X-ray scattering (SAXS) is a powerful technique, particularly useful for conformationally flexible systems (Receveur-Brechot \& Durand, 2012) and especially when partial structural information is available. Furthermore, coupling SAXS to an inline separation technique such as size-exclusion chromatography (SEC) has greatly improved the utility of the measurement for complex systems such as protein:detergent complexes (PDCs) (Mertens \& Svergun, 2010). The method allows separation of the PDC from free detergent micelles, thus providing scattering information of the solubilized system components (Ryan et al., 2018). Advances in ab initio and rigid body modelling methods combined with accurate computation of 
scattering curves from atomic coordinates can provide reliable models to gain insight into the structural conformation of proteins in solution (Koutsioubas, 2017; Koutsioubas et al., 2013). Importantly, these modelling approaches can be used to characterize the structure and function of proteins with significant flexibility, where information on alternate states of the system are difficult or even impossible to obtain by higher resolution methods. The snapshot conformational state captured in the structural models obtained by crystallography can be used in hybrid approaches to further probe conformations of the molecule using data from SAXS and other methods. In this study, we investigate the conformational states of EptA in DDM micelles and DPC micelles using SECSAXS and intrinsic fluorescence quenching. Intrinsic fluorescence quenching experiments on mutants of the enzyme where tryptophan residues have been replaced allow us to further probe the local environment of both the soluble and transmembrane domains of EptA as a function of the detergent environment.

The SEC-SAXS study successfully deconvolutes the scattering data of the EptA detergent complex, models the detergent corona around the protein using two independent methods and compares the generated models in different detergent environments. The fluorescence study identifies specific structural regions of the enzyme responsible for conformational changes which localize the interdomain flexibility to the bridging helix linking the soluble and transmembrane domains, and suggests that this flexibility plays a critical role in the catalytic function of the enzyme. This multifaceted biophysical approach not only provides unique information regarding the conformational flexibility of EptA, but also facilitates procedures to study other membrane proteins aimed at understanding the role of conformational flexibility for function.

\section{Methods}

\subsection{Plasmid construction for EptA tryptophan mutants}

EptA tryptophan mutants were made by two rounds of splicing by overlap extension polymerase chain reaction (SOE PCR) where the tryptophan residues at positions 126, 148 and 207 were replaced with phenylalanines. In the first round of SOE PCR, separate fragments with the W126F and W207F mutations were amplified from wild type eptA using primers containing these mutations. In the second round of SOE PCR, these fragments were used as a template by which overlapping complementary ends allowed for annealing and formation of the entire gene, 'sewing' the fragments together to create a single mutant construct (W126F) and a double mutant construct (W126F/W207F). This two-step process was repeated to introduce the $\mathrm{W} 148 \mathrm{~F}$ mutation, using the $\mathrm{W} 126 \mathrm{~F}$ mutant as the template to make the double mutant (W126F/ $\mathrm{W} 148 \mathrm{~F}$ ) and using the $\mathrm{W} 126 \mathrm{~F} / \mathrm{W} 207 \mathrm{~F}$ double mutant as the template to make the triple mutant (W126F/W207F/W148F). Table S1 of the supporting information lists the primers used during each round of SOE PCR. The mutants were cloned into the NcoI and BamHI sites of the pET28a (+) vector. Each constructed mutant plasmid was verified by colony PCR and restriction endonuclease digest as well as DNA sequencing.

For expression in Neisseria meningitidis cells, the mutants were excized from the respective pET28a (+) vectors using the restriction enzymes XbaI and SalI and cloned into the XbaI and SaI sites of a neisserial shuttle vector. This vector consists of the gonococcal cryptic fragment from pYT250 (Tzeng et al., 2002) ligated into the low copy plasmid pHSG576 containing a chloramphenicol resistance marker and two different origins of replication (Takeshita et al., 1987), enabling replication in both Escherichia coli and Neisseria species. It also contains an ompR promoter to drive downstream gene expression and a synthetically produced multiple cloning site. Successful cloning of both of mutants was confirmed by colony PCR and restriction endonuclease digest.

\subsection{Polymyxin B sensitivity assay}

Polymyxin B sensitivity was determined via E-strip testing. Following natural transformation (Janik et al., 1976) of the shuttle vectors into strain NMB $\triangle e p t A$, overnight growth on gonococcal base agar supplemented with $0.4 \%(w / v)$ D-glucose solution and $0.68 \mathrm{~m} M$ iron(III) nitrate (GCGF) of each strain was collected using a sterile loop and inoculated in $5 \mathrm{ml}$ of GC broth $[1.5 \%(w / v)$ special peptone, $0.4 \%$ dipotassium phosphate, $0.1 \%$ potassium dihydrogen phosphate and $0.5 \%$ sodium chloride]. The broth suspensions were centrifuged at $60 \mathrm{~g}$ for $2 \mathrm{~min}$ to remove clumps and standardized to an $\mathrm{OD}_{560}$ of 0.4. Standardized suspensions were then spread onto GCGF plates in volumes of $100 \mu$ l. GCGF plates were allowed to airdry for 10 min and polymyxin B E-strips (BioMérieux) were then placed in the centre of the plates. GCGF plates were then incubated overnight at $37^{\circ} \mathrm{C}$ with $5 \% \mathrm{CO}_{2}$ and the zone of inhibition was reported.

\subsection{Protein expression and purification}

EptA for SEC-MALS and SEC-SAXS experiments was expressed using the pTrc99A expression vector as detailed in the work by Anandan et al. (2017). The mutants used for the tryptophan fluorescence experiments were expressed using the pET28a (+) expression vector designed as described above. All proteins were purified in DDM micelles and DPC micelles as detailed in the work by Anandan et al. (2017). The vectors containing the WT eptA gene with a C-terminal hexahistidine tag were expressed in E. coli strain BL21 (DE3) pLys while the mutants were expressed in E. coli BL21 (DE3) Rosetta 2. The cultured E. coli cells were harvested by centrifugation ( $12290 \mathrm{~g}$ for $1 \mathrm{~h}$ at $\left.4^{\circ} \mathrm{C}\right)$ and lysed in $50 \mathrm{~m} M$ phosphate buffer pH 7.5 containing $300 \mathrm{mM} \mathrm{NaCl}$ and $1 \mathrm{~m} M$ PMSF using an Emulsiflex C5 high-pressure homogenizer (Avestin Emulsiflex C5). The membranes from the lysate were isolated by ultracentrifugation (185511 $\mathrm{g}$ for $1 \mathrm{~h}$ at $4^{\circ} \mathrm{C}$ ). EptA was solubilized and separated from the membranes by incubating the lysate for $4 \mathrm{~h}$ with $1 \%$ detergent (DDM or DPC) in $50 \mathrm{~m} M$ phosphate buffer $\mathrm{pH} 7.5$ containing 
$300 \mathrm{~m} M \mathrm{NaCl}, 10 \mathrm{~m} M$ imidazole and $1 \mathrm{~m} M$ PMSF followed by ultracentrifugation ( $185511 \mathrm{~g}$ for $1 \mathrm{~h}$ at $4^{\circ} \mathrm{C}$ ).

Metal-chelating chromatography and size-exclusion chromatography were used for EptA purification. The supernatant obtained from ultracentrifugation was applied to a preequilibrated HisTrap crude extract chelating affinity column (GE Healthcare) with the binding buffer [50 $\mathrm{m} M$ sodium phosphate $\mathrm{pH} 7.5,300 \mathrm{~m} M$ sodium chloride, $20 \mathrm{~m} M$ imidazole and $3 \times \mathrm{CMC}$ detergent $(0.023 \% \mathrm{DDM}$ or $0.14 \% \mathrm{DPC})]$. The bound protein was then eluted with the elution buffer [50 $\mathrm{m} M$ sodium phosphate $\mathrm{pH}$ 7.5, $300 \mathrm{~m} M$ sodium chloride, $500 \mathrm{mM}$ imidazole and $3 \times$ CMC detergent $(0.023 \%$ DDM or $0.14 \%$ DPC)] using an ÅKTApurifier FPLC system (GE Healthcare). The peak corresponding to EptA was pooled, buffer exchanged into $50 \mathrm{~m} M$ sodium phosphate $\mathrm{pH} 7.5,150 \mathrm{~m} M$ sodium chloride and $3 \times \mathrm{CMC}$ detergent $(0.023 \% \mathrm{DDM}$ or $0.14 \%$ DPC) using a PD-10 desalting column (GE Healthcare) and applied to a size-exclusion chromatography column (Superdex 200 10/30, GE Healthcare) equilibrated with $50 \mathrm{~m} M$ HEPES $\mathrm{pH} 7.0,100 \mathrm{~m} M \mathrm{NaCl}$ and $3 \times \mathrm{CMC}$ detergent (0.023\% DDM or $0.14 \%$ DPC). Fractions of EptA from the elution peak were concentrated using centrifugal filter units (Vivaspin 20 MWCO 100000 , GE Healthcare for DDM purified EptA and Vivaspin 20 MWCO 50 000, GE Healthcare for DPC purified EptA) to 7 or $2 \mathrm{mg} \mathrm{ml}^{-1}$ for protein used in SEC-SAXS work and in fluorescence work, respectively, as determined by the absorbance at $280 \mathrm{~nm}$ and calculated molar extinction coefficient $\left(73980 \mathrm{M}^{-1} \mathrm{~cm}^{-1}\right.$ for WT EptA, $62980 \mathrm{M}^{-1} \mathrm{~cm}^{-1}$ for the double mutant and $57480 \mathrm{M}^{-1} \mathrm{~cm}^{-1}$ for the triple mutant).

\subsection{Circular dichroism spectropolarimetry}

The folded state of WT and mutant EptA was confirmed following buffer exchange into $20 \mathrm{~m} M$ sodium phosphate $\mathrm{pH}$ $7.0,3 \times$ CMC detergent $(0.023 \%$ DDM or $0.14 \%$ DPC) with a $5 \mathrm{ml}$ HisTrap Desalting Column using an ÅKTApurifier FPLC system and diluted to a concentration of $0.1 \mathrm{mg} \mathrm{ml}^{-1}$. The farUV spectral region was measured at $20^{\circ} \mathrm{C}$ on a circular dichroism (CD) spectrophotometer (Jasco J-720) using a $1 \mathrm{~mm}$ path length quartz cuvette. Data were collected every $1 \mathrm{~nm}$ in the 190-260 nm wavelength region with an integration time of $1 \mathrm{~s}$ per step and three accumulations. The $\mathrm{CD}$ spectra were collected in triplicate, corrected for the buffer baseline and the signal-to-noise ratio was improved using a SavitzkyGolay filter with the minimum convolution width (of five data points) using Spectra Analysis software (version 1.53.07 for Windows 95/NT, Jasco Corp.).

\subsection{SEC-MALS data collection and analysis}

SEC-MALS analysis of EptA in DDM and DPC micelles was performed using a 10/300GL Superdex 200 column (GE Healthcare) coupled to a Viscotek TDA tetra detector array (Malvern). The SEC-MALS system was equilibrated with $50 \mathrm{~m} M$ HEPES $\mathrm{pH} 7.0,100 \mathrm{~m} M \mathrm{NaCl}$ and $3 \times \mathrm{CMC}$ detergent (0.023\% DDM or $0.14 \%$ DPC) to obtain a flat baseline for all the detectors. The instrument was then calibrated against
$100 \mu \mathrm{l}$ of $1 \mathrm{mg} \mathrm{ml}^{-1}$ bovine serum albumin prepared in $50 \mathrm{mM}$ HEPES $\mathrm{pH} 7.0,100 \mathrm{mM} \mathrm{NaCl}$ and $3 \times \mathrm{CMC}$ of respective detergent $(0.023 \%$ DDM or $0.14 \%$ DPC). The integrity of EptA purified in DDM micelles and DPC micelles was analysed by injecting $100 \mu \mathrm{l}$ of the protein at a concentration of $1 \mathrm{mg} \mathrm{ml}^{-1}$ into the SEC-MALS system and flowing the sample at $0.3 \mathrm{ml} \mathrm{min}{ }^{-1}$. Viscotek's OmniSEC software was used for data collection and analysis. The multi-detector copolymer method was used for analysis and molecular weight determination of protein:detergent complex (PDC) and micelles eluted from the column (Pollock et al., 2012). Peak limits for PDC and detergent micelles were manually defined and the $\mathrm{d} n / \mathrm{d} c$ values for protein $\left(0.185 \mathrm{ml} \mathrm{g}^{-1}\right)$ and detergents $\left(0.1608 \mathrm{ml} \mathrm{g}^{-1}\right.$ for DDM and $0.1398 \mathrm{ml} \mathrm{g}^{-1}$ for DPC) were used for the molecular mass calculations.

\subsection{SEC-SAXS data collection and analysis}

Inline SEC-SAXS was performed at the SAXS/WAXS beamline at the Australian Synchrotron. Protein samples were subjected to size-exclusion chromatography using a S200 10/300 increase column (GE Healthcare) attached to a Shimadzu HPLC system and passing through a capillary within a SAXS coflow cell (Kirby et al., 2016; Ryan et al., 2018). The column was pre-equilibrated with $50 \mathrm{~m} M$ HEPES $\mathrm{pH}$ 7.0, $100 \mathrm{~m} M$ sodium chloride, $0.1 \%$ sodium azide and appropriate detergent concentration $(4 \times \mathrm{CMC}$ of DDM or $3 \times \mathrm{CMC}$ of DPC). $50 \mu \mathrm{l}$ of $7.0 \mathrm{mg} \mathrm{ml}^{-1}$ EptA purified in DDM micelles or $7.0 \mathrm{mg} \mathrm{ml}^{-1}$ EptA purified in DPC micelles was injected onto the column and eluted at 0.2 and $0.4 \mathrm{ml} \mathrm{min}^{-1}$, respectively. SAXS data were acquired using X-rays at an energy of $12 \mathrm{kEV}$ and a flux of $3.1 \times 10^{12}$ photons s $^{-1}$. As data were collected on two different occasions, EptA in DPC SAXS data were collected on a Dectris PILATUS $1 \mathrm{M}$ detector at $2.5 \mathrm{~m}$ (providing the $q$ range $0.005-0.3 \AA$ ) and EptA in DDM SAXS data were collected using a Dectris PILATUS 2M detector at $2 \mathrm{~m}$ (providing the $q$ range $0.01-0.5 \AA$ ). The frames were collected continuously and integrated every $5 \mathrm{~s}$. The SAXS data collection and data reduction statistics as well as details of the software used for data analyses are given in Table S2. The program SCATTERBRAIN 2.82, developed at the Australian Synchrotron, was used to reduce the two-dimensional scattering patterns to one-dimensional profiles followed by the ATSAS 3.0 software suite for post-experiment data analysis (Franke et al., 2017). The integrated errors from the output of SCATTERBRAIN were of two standard errors and had to be divided by two to meet the standard definition of $\chi^{2}$ to accurately reflect the standard of the fit (Ryan et al., 2018). CHROMIXS (Panjkovich \& Svergun, 2018) was then used to subtract buffer frames (frames 100-155) from the PDC elution peak frames to generate the SAXS curve for further analysis. The radius of gyration $\left(R_{\mathrm{g}}\right)$ was measured for frames across the protein elution peak, the traces of which were derived from the SAXS/WAXS pipeline, which is based on AUTORG, but is more sensitive to noise in the low $q$ range. Frames from 391 to 409 for EptA in DDM micelles and frames from 364 to 389 for EptA in DPC micelles were selected for 
analysis. PRIMUS (Konarev et al., 2003) was used for plotting and inspecting the CHROMIXS-generated SAXS curve, $A U T O R G$ was used for generating $I(0)$ and $R_{\mathrm{g}}, D A T G N O M$ was used to generate the $P(r)$ functions (Franke et al., 2017), and DATCMP (Franke et al., 2017; Petoukhov et al., 2012) was used to compare the similarity of scattering curves. The dimensionless Kratky plot was calculated according to the work by Durand et al. (2010) using the value determined by Guinier analysis, and $P(r)$ functions were computed from the scattering curves by an indirect transform method in GNOM (Svergun, 1992). The molecular weight was calculated from $I_{0}$ using the method by Mylonas \& Svergun (2007), with a partial specific volume calculated using the weight average composition of the PDC from the MALS data, a partial specific volume for EptA calculated from the sequence using $\mathrm{MulCH}$ (Whitten et al., 2008), and the quoted partial specific volumes for the DDM and DPC from Anatrace (https://www. anatrace.com). The electron density of the solvent and PDC was calculated from the composition and elemental makeup. The concentration of the PDC was calculated from the in-line UV absorbance measurements, and an A280 nm extinction coefficient for the PDC was calculated using PROTPARAM.

\subsection{Model building}

2.7.1. MEMPROT and DADIMODO. MEMPROT (release 2.2) (Perez \& Koutsioubas, 2015) was used to generate hybrid models of the protein:detergent complexes, with the highresolution structure of EptA (PDB entry 5fgn; Anandan et al., 2017) used to model the protein component and the detergent corona (DDM or DPC) encircling the transmembrane region of EptA modelled as dummy atoms. The crystal structure lacked the first eight residues at the $\mathrm{N}$ terminus and the hexahistidine tag at the $\mathrm{C}$-terminus. The crystal structure lacking the above-mentioned residues was oriented semimanually using the $P y M O L$ software following the instructions from the MEMPROT package such that the transmembrane domain aligned parallel to the $z$ axis. The elevation and orientation of the structure with respect to the $x y$ plane was adjusted (Perez \& Koutsioubas, 2015) in such a way that the aromatic residues forming the aromatic belt of EptA (Anandan et al., 2017) aligned perpendicular to the $z$ axis. MEMPROT generated models of the detergent corona around the EptA structure were made by sampling five geometrical parameters, namely ' $a$ ' (height of the detergent torus), ' $b$ ' [cross section of the torus with major (b.e) and minor (b/e) axes], ' $t$ ' (thickness of the hydrophilic shell), ' $e$ ' (ellipticity) and ' $\omega$ ' (the angle of rotation of the corona around the $z$ axis) to fit the experimental SAXS curve. The inner hydrophobic and outer hydrophilic regions of the corona were defined by factoring in the electron densities of the corresponding detergents. Electron density used for modelling the DDM corona was $0.277 \mathrm{e}^{-3}$ for the hydrophobic portion and $0.520 \mathrm{e}^{-3}$ for hydrophilic portion of the DDM molecules, whereas the electron density for modelling DPC corona was $0.277 \mathrm{e} \AA^{-3}$ for the hydrophobic portion and $0.490 \mathrm{e} \AA^{-3}$ for the hydrophilic portion (Lipfert et al., 2007). The program

Table 1

Statistics for the small-angle X-ray scattering data analysis and modelling of EptA in different detergent micelles.

Structure parameters EptA in DDM micelles EptA in DPC micelles

Gunier analysis

I(0) $\left(\mathrm{cm}^{-1}\right)$

$R_{\mathrm{g}}(\AA)$

$s R_{\mathrm{g}}$ limits

$P(r)$ analysis

$I(0)\left(\mathrm{cm}^{-1}\right)$

$R_{\mathrm{g}}$ from $P(r)(\AA)$

$D_{\text {max }}(\AA)$

$s$ range $\left(\AA^{-1}\right)$

$0.013 \pm 1.9 \times 10^{-4}$

$41.96 \pm 0.71$

$0.60 \pm 1.30$

$0.013 \pm 0.9 \times 10^{-4}$

$41.45 \pm 0.01$

128.9

$0.01-0.2$

Molecular weight estimation

SEC-SAXS (kDa)

200.25 to 218.51

SEC-MALS (kDa)

$160 \pm 8.0$

Atomistic modelling and model-fitting results

MEMPROT (detergent torus dimensions)

Height $(a)(\AA)$

Cross section $(\AA)$

Major axis $(b . e)$

Minor axis (b/e)

Ellipticity

Thickness of th
layer $(t)(\AA)$

Angle of rotation of corona 110

around $z$ axis $(\omega)\left(^{\circ}\right)$

$\chi^{2}$

DADIMODO

No. of models generated N/A

$\chi^{2}$ range for 5 models N/A

MPBuilder (detergent corona dimensions)

No. of detergent molecules 225

added using $P A C K M O L$

routine

Bilayer height $(\AA)$

Cross section $(\AA)$

Major axis

Minor axis

Ellipticity

45

64

$$
37.5
$$

1.7
$0.0052 \pm 3.3 \times 10^{-5}$
$45.78 \pm 0.40$
$0.40 \pm 1.35$
$0.0052 \pm 2.8 \times 10^{-5}$
$48.14 \pm 0.01$
171.06
$0.005-0.21$

125.51 to 136.37

$109 \pm 3.4$

CORAL

Flexible residues $\quad 1-7,211-230,544-550 \quad 1-7,211-230,544-550$

$s$ range for modelling $\left(\AA^{-1}\right) \quad 0.01-0.32 \quad 0.006-0.288$

$\chi^{2}, P$ value $\quad 1.18,0.00 \quad 1.84,0.16$

EOM

Flexible residues $\quad 1-7,211-230,544-550 \quad 1-7,211-230,544-550$

$s$ range for modelling $\left(\AA^{-1}\right)$

$\chi^{2}, P$ value

$0.01-0.10$

$0.01-0.10$

$0.99,0.85$

calculated parameter values to generate models that best fitted the experimental curve. The agreement between the model generated and the SAXS experimental data is assessed by the $\chi^{2}$ value generated by CRYSOL (Svergun et al., 1995) (Table 1).

To improve the modelling guided by the SAXS data and to optimize the relative positioning of the soluble and transmembrane domains of EptA, the program DADIMODO (Evrard et al., 2011) was used. The MEMPROT generated model was used as a starting structure for the DADIMODO genetic algorithm. Both the soluble and transmembrane domain structures were kept rigid while the region linking 
both the domains, namely the bridging helix and the extended loop region, were defined as flexible regions for the program.

2.7.2. MPBUILDER and CORAL. All-atom models of the protein:detergent complexes were generated and refined against the SAXS data using MPBUILDER (Molodenskiy et al., 2021) coupled with the rigid-body modelling program CORAL (Petoukhov et al., 2012). As the crystal structure lacked the first eight residues and the histidine tag, these were incorporated into the initial model prior to further modelling. An initial approximation of the number of bound detergent molecules was made based upon the SEC-MALS analysis, and 160 DPC or 225 DDM monomers were added to the transmembrane domain of EptA (PDB entry 5fgn) using the PACKMOL routine (Martínez et al., 2009) implemented in $M P B U I L D E R$. The transmembrane domain was pre-oriented for membrane insertion using the PPM server (Lomize et al., 2012) located at the OPM database website (http:// opm.phar.umich.edu). The soluble and detergent-bound transmembrane domains were used as input rigid bodies in $C O R A L$, and the position of the soluble domain relative to the transmembrane domain refined against the SAXS data, with the linker region between the domains ( 34 residues) and terminal residues defined as flexible dummy residues.

2.7.3. Flexibility analysis by the Ensemble Optimization Method. Using the low-angle range $(q<0.1 \AA)$ of the SAXS data, corresponding to the overall shape of the protein: detergent complexes only, the Ensemble Optimization Method (EOM) (Tria et al., 2015) was applied to obtain an estimate of the intrinsic flexibility of the EptA protein solubilized in DDM or DPC detergents. The same rigid bodies as described for the MPBUILDER/CORAL modelling were used as input for the generation of a pool of 10000 models for each detergent type, with the inter-domain linker and termini defined as flexible and the soluble domain allowed to sample all possible conformations. Conformations generated that formed steric clashes with the detergent shell (van der Waals cut-off $<0.4 \mathrm{~A}$ ) were discarded, leaving approximately 5000 conformations for the final pool and subsequent analysis for both DDM and DPC EptA complexes. Scattering intensities for each conformer in each pool were computed using CRYSOL (Svergun et al., 1995). The intensities corresponding to the pool were then subjected to the genetic algorithm routine $G A J O E$ within $E O M$, and a subset of conformations that best fit the experimental data were selected through a series of random selection and elitism steps. The flexibility assessment was then performed on size distributions corresponding to the radii of gyration of the corresponding conformers selected and compared with that of the pool. The statistic $R_{\text {flex }}$ (Tria et al., 2015) was calculated for both the selected ensemble and the pool, where $R_{\text {flex }}$ approaching $100 \%$ indicates a fully flexible system of random conformers.

\subsection{Intrinsic tryptophan fluorescence measurements}

Intrinsic fluorescence was monitored using an EnSpire Multimode plate-reader (Perkin Elmer). Sample excitation was carried out at $295 \mathrm{~nm}$ (100 flashes; $<8 \mathrm{~nm}$ bandwidth) in order to selectively excite tryptophan residues and emission spectra were measured from 315 to $405 \mathrm{~nm}$ at $1 \mathrm{~nm}$ intervals. Protein samples at $12 \mu M$ concentration in $50 \mathrm{~m} M$ HEPES $\mathrm{pH}$ $7.0,50 \mathrm{mM} \mathrm{NaCl}$ and $3 \times \mathrm{CMC}$ detergent $(0.023 \% \mathrm{DDM}$ or $0.14 \%$ DPC) to a final volume of $100 \mu \mathrm{l}$ were gradually quenched by the addition of small aliquots $(1$ or $2 \mu \mathrm{l})$ of $4 M$ potassium iodide also containing $0.2 \mathrm{~m} M$ sodium thiosulfate to prevent the formation of triiodide $\left(\mathrm{I}_{3}^{-}\right)$. Non-quenched samples were maintained at the same ionic strength by including equivalent volumes of potassium chloride. For the intrinsic fluorescence of denatured protein, samples also contained $4 M$ guanidine hydrochloride as a denaturant and $0.2 M \beta$-mercaptoethanol to maintain reducing conditions throughout the experiment. All samples were incubated for $15 \mathrm{~min}$ at $20^{\circ} \mathrm{C}$ in a UV-Star half-area microplate (Greiner Bio-One). Spectra were recorded from three replicate protein wells and corrected by subtracting measurements from three replicate protein-free wells of the same volume and equivalent salt and buffer compositions.

Data obtained from quenching experiments were fitted to equation (1) in order to determine the Stern-Volmer constant $\left(K_{\mathrm{SV}}\right)$, where $\left[\mathrm{I}^{-}\right]$corresponds to the iodide concentration:

$$
F_{0} / F=K_{\mathrm{SV}} \mathrm{X}\left[\mathrm{I}^{-}\right]+1 .
$$

The relative fluorescence intensity of unquenched to quenched protein $\left(F_{0} / F\right)$ was obtained from triplicate spectra by dividing the maximum protein fluorescence emission in the presence of $\mathrm{KCl}$ by the maximum protein fluorescence emission with an equivalent concentration of KI. This corrects for changes to the ionic strength and volume of samples with each addition of the quenching or non-quenching salt (i.e. KI and $\mathrm{KCl}$, respectively). Equation (1) was fitted to the linear regions of the quenching data using linear regression in the GraphPad Prism software (version 5.02 for Windows, GraphPad Software Inc.).

\section{Results}

3.1. Characterization of the protein:detergent complex by SEC-MALS analysis

EptA purified in DDM micelles and DPC micelles were analysed by SEC-MALS in order to establish that the protein was monodisperse in different detergent micelles and amenable to SAXS studies. Figs. S1 $(a)$ and S1 $(b)$ of the supporting information display the overlayed chromatograms obtained from the UV absorption at $280 \mathrm{~nm}$ and refractive index (RI) signal superimposed on the right-angle scattering signal for EptA in DDM micelles and DPC micelles, respectively. EptA in DDM micelles eluted at $14.11 \mathrm{ml}$ as a single peak as detected by the UV absorbance while the RI signal displayed an additional peak at $15.9 \mathrm{ml}$ corresponding to free DDM micelles. A curvature across the elution peak was evident for EptA in DDM micelles suggesting a possible dynamic equilibrium or concentration-dependent association. While preliminary SEC-SAXS experiments altering protein/detergent concentration ratios were carried out as an optimization 
process (data not presented) the current ratio of [EptA]:[DDM] of $7 \mathrm{mg} \mathrm{ml}^{-1}: 4 \times \mathrm{CMC}$ was chosen for the final SEC-SAXS analysis as the sample in this condition behaves more uniformly and mondisperse. EptA in DPC micelles eluted at $14.41 \mathrm{ml}$ and the free DPC micelles eluted at $16.74 \mathrm{ml}$. Although a small overlap between the PDC peak and detergent micelle peak was observed for both samples, the peak boundaries were carefully set to minimize the overlap before data analysis was undertaken. The average molecular weight $(\mathrm{MWt})$ of the EptA:DDM complex estimated by RI was $160.3 \pm 8.0 \mathrm{kDa}$ and the calculated MWt of EptA was $58.31 \mathrm{kDa}$. For the EptA:DPC complex, the average MWt was estimated to be $109.0 \pm 3.4 \mathrm{kDa}$ and that of EptA alone was estimated at $62.85 \mathrm{kDa}$. The molecular weight estimation of EptA in DDM micelles was slightly lower than the theoretical value, estimated from PROTPARAM (Gasteiger et al., 2005) $(61.40 \mathrm{kDa})$ while the estimation of EptA in DPC micelles was closer to the theoretical value. Based on these results the average numbers of DDM and DPC molecules associated with the protein:detergent complex were estimated to be 200 and 123 , respectively.

\subsection{Conformational analyses of the protein:detergent complexes by SEC-SAXS}

The conformation of EptA purified in the two aforementioned detergent micelles was analysed by SEC-SAXS at the Australian Synchrotron. For both detergent systems, the elution profile from the S200 10/300 increase SEC column revealed a PDC peak slightly overlapped with the detergent micelle peak; however, the separation was sufficient to provide unambiguous SAXS signals from the PDC and detergent micelle species as shown by the similarity of the extracted SAXS parameters across the peak maxima (Fig. S2 of the supporting information). The molecular weight for the PDC from the SAXS signal was determined as described by Mylonas \& Svergun (2007), where the partial specific volume was estimated from the MALS data, and the protein concentration was determined using inline A280 nm absorbance measurements, and the extinction coefficient was obtained from the EptA sequence using PROTPARAM (Gasteiger et al., 2005). Additionally, a comparable result was obtained through a Bayesian approach for molecular weight determination, carried out using the ATSAS software (Franke et al., 2017; Hajizadeh et al., 2018). Molecular weight analyses of the PDC species for frames selected from the SEC-SAXS peak were provided by the Bayesian molecular weight calculation in ATSAS. The values ranged from 200 to $218.5 \mathrm{kDa}$ for the EptA:DDM complex and from 125.5 to $136.37 \mathrm{kDa}$ for the EptA:DPC complex [Fig. S2(a)]. Guinier analyses of the PDC peak for the sample in DPC micelles exhibited less variation in $R_{\mathrm{g}}$ across the peak than was observed for the sample in DDM micelles [Fig. S2(b)]. While these values for molecular weight differ somewhat from those determined by the SEC-MALS analyses, other examples show deviations of 10-20\% (Gimpl et al., 2016; Mirandela et al., 2018) and are well within the accuracy expected from SAXS determination of molecular weight (Mylonas \& Svergun, 2007). Additionally, the differences in the concentrations of the protein and detergent within each experiment may account for the variation observed in the number of detergent molecules by SECMALS and by SEC-SAXS.

The averaged, buffer-subtracted SAXS profile of the EptA:DDM complex and the EptA:DPC complex are presented in Fig. 2(a). Guinier analysis of both the
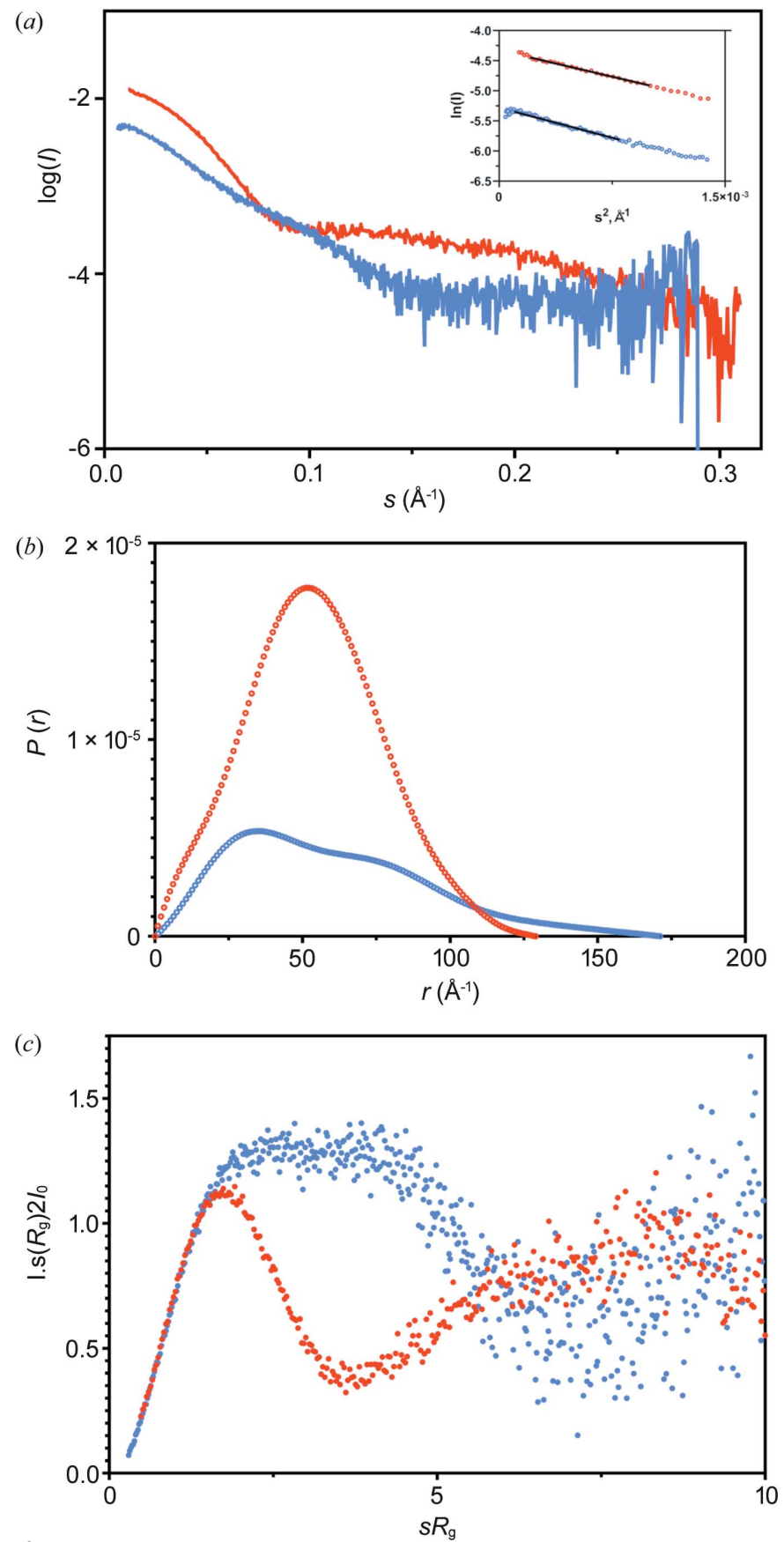

Figure 2

SEC-SAXS results for the EptA:DDM complex and EptA:DPC complex. (a) $I(s)$ versus $s$, as $\log$-linear plots with the inset showing the Guinier fits (black line) laid on the experimental data (open symbols). (b) Profiles for the distance distribution function, $P(r)$, in arbitrary units versus $r$ in ångströms for the data shown in $(a)$, normalized for ease of comparison. (c) Dimensionless Kratky plots for the data in $(a)$. 
EptA:DDM complex and the EptA:DPC complex revealed linear Guinier plots [Fig. 2(a) inset] without evidence of aggregation/interparticle interaction in the samples. For the EptA:DDM complex an $R_{\mathrm{g}}$ value of $42.0 \pm 0.7 \AA$ was determined and the distance distribution function, $P(r)$, showed a single bell-shaped distribution with a maximal dimension $\left(D_{\max }\right)$ of $129 \AA$, suggesting a compact globular structure. For the EptA:DPC complex, an $R_{\mathrm{g}}$ value of $45.8 \pm 0.4 \AA$ was determined. The $P(r)$ showed a peak with an extended tail, characteristic of an elongated particle shape, and a $D_{\max }$ of $171 \AA$. The extracted SAXS parameters and the significant differences in the shape of the distance distributions clearly show the EptA:DPC complex to be larger and more extended than the EptA:DDM complex (Kikhney \& Svergun, 2015). The symmetric bell-shaped $P(r)$ distribution of the EptA:DDM complex and extended $P(r)$ distribution for the EptA:DPC complex are shown in Fig. 2(b).

The dimensionless Kratky plot (assessing the globularity and flexibility of the protein) shows notable differences in the intermediate $s$ range for EptA in different detergent systems [Fig. 2(c)]. The EptA:DDM complex exhibits a bell-shaped curve, with maximum of 1.147 at $1.9 s R_{\mathrm{g}}$ indicating a folded/ globular shaped molecule with limited flexibility. The EptA:DPC complex shows a maximum of 1.4 at $2.8 s R_{\mathrm{g}}$ with an additional shoulder at the intermediate $s$ region and converging at higher angles, suggesting that this complex is both more elongated and potentially more flexible than the EptA:DDM complex (Durand et al., 2010; Rambo \& Tainer, 2011).

\subsection{MEMPROT and DADIMODO modelling results}

The SAXS profile of a PDC comprises signal from both the detergent solubilized protein and from the protective amphiphilic corona formed by detergent molecules. In order to gain insight into the conformation adopted by the protein in different detergent environments, models of the PDC consistent with the experimental SAXS curves were generated (Table 1).

MEMPROT (Pérez \& Koutsioubas, 2015) was employed to model the detergent assemblies as course grained dummy atoms around the hydrophobic region of the EptA crystal structure (PDB entry 5fgn; Anandan et al., 2017). For the EptA:DDM complex the best fitting models built an elliptical DDM torus around the EptA molecule [Fig. 3(a) panel (i)] with an inner hydrophobic region $31 \AA$-thick and an outer hydrophilic region $4.8 \AA$ A-thick [Fig. S3(a) panels (ii) and (iii)]. The derived SAXS profile for this model closely matched the experimental SAXS curve $\left[\chi^{2}=1.2\right.$; Fig. $3(a)$ panel (ii)]. MEMPROT estimated $235 \pm 37 \mathrm{DDM}$ molecules in the detergent corona which corresponded well with the estimate obtained by SEC-MALS (200 DDM molecules).

For the PDC in DPC the MEMPROT models generated [Fig. 3(b) panel (i)] and their corresponding SAXS profile departed considerably from the experimental curve $\left[\chi^{2}>3.5\right.$; Fig. 3(b) panel (ii)]. Based on the primary SAXS analysis discussed above, the possible conformational change to a more extended structure was investigated through further refinement of the MEMPROT EptA:DPC complex model using DADIMODO (Evrard et al., 2011). DADIMODO uses a genetic algorithm approach to sample domain positions and select the best fitting model conformations using SAXS data. The MEMPROT generated model of the EptA:DPC complex resulted in a DPC corona with a hydrophobic inner region of $24.7 \AA$ and a hydrophilic outer region of $3.7 \AA$ [Fig. S3 $(b)$ panels (ii) and (iii)]. The number of DPC detergent molecules modelled in the detergent corona was $190 \pm 3$; however, as the EptA model is constrained to the crystallographic coordinates, if the system is indeed dynamic this estimate may not be reliable and can be taken only as a rough guide. This model of EptA in the DPC torus was used as input to DADIMODO with the bridging helix and extended loop (residues 174-230) defined as the flexible region of the structure. DADIMODO generated five different models [a representative model is shown in Fig. 3(b) panel (iii)] which generated calculated SAXS profiles that fit well with the experimental SAXS data $\left[\chi^{2}=1.05-1.07\right.$; Fig. 3(b) panel (iv) $]$. All five models were similar, displaying the soluble domain rolled away from the transmembrane domain with an increased distance between the centre of mass of the domains when compared with the crystal structure of EptA.

The results of this analysis provide experimental and structural evidence that the protein in the two different detergent micelle environments adopts different conformations with the orientation of the two protein domains varying in a detergent-dependent manner. Interestingly, the altered conformation adopted by EptA in DPC micelles resembled the in silico molecular dynamics simulations of the enzyme in a lipid bilayer (Anandan et al., 2017) as a more open structure compared with that observed in the DDM micelles.

\subsection{MPBuilder and CORAL modelling results}

In a parallel approach to the model determination of the EptA complexes with dummy atoms representing detergent, hybrid models were computed using the MPBuilder algorithm (Molodenskiy et al., 2021) combined with the programs PACKMOL (Martínez et al., 2009) and CORAL (Petoukhov et al., 2012). This procedure allowed for the generation of allatom models of the complexes from the crystal structure of EptA and both DDM and DPC detergent micelles. In addition, this approach modelled the inter-domain loop, allowing for rearrangement of the soluble and transmembrane domains, as well as the terminal residues not observed in the EptA crystal structure. Qualitatively, the models generated for both detergent complexes [Figs. 4(a) and 4(b) panel (i)] are similar to those generated from the hybrid ab initio approaches employed by MEMPROT and DADIMODO. The best fitting models for the DDM and DPC complexes again demonstrated different conformations, with a compact structure observed for EptA in the presence of DDM and a significantly more extended structure observed in the presence of DPC. 
(a)

(i)

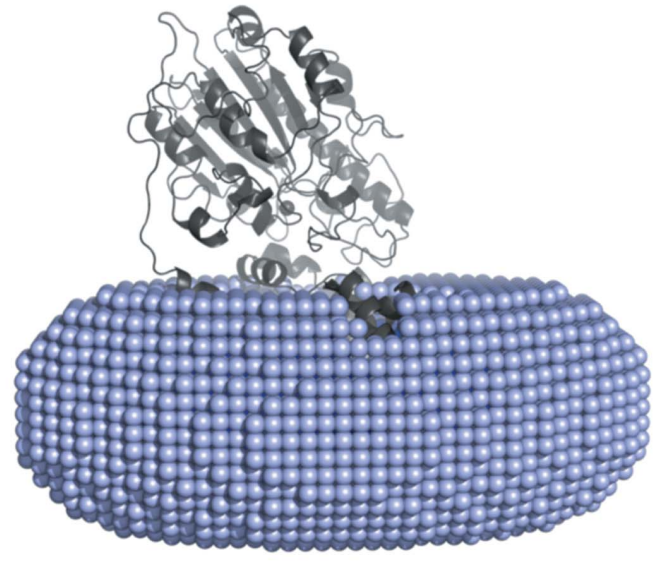

(b)

(i)

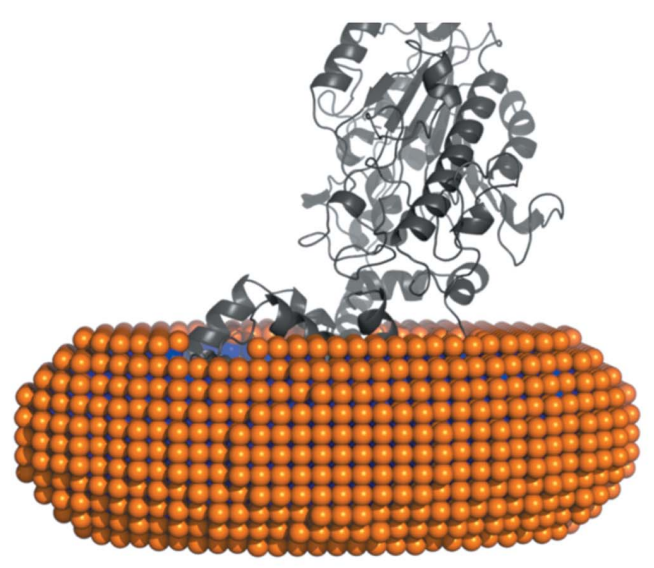

(iii)

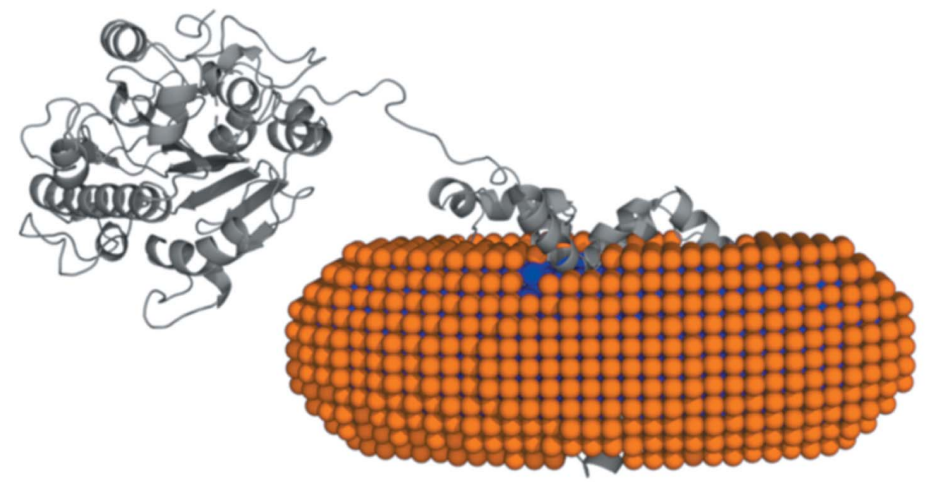

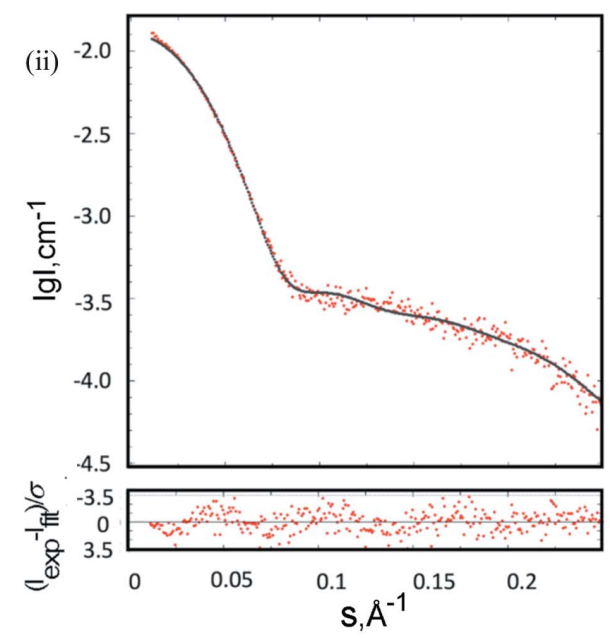

(ii)
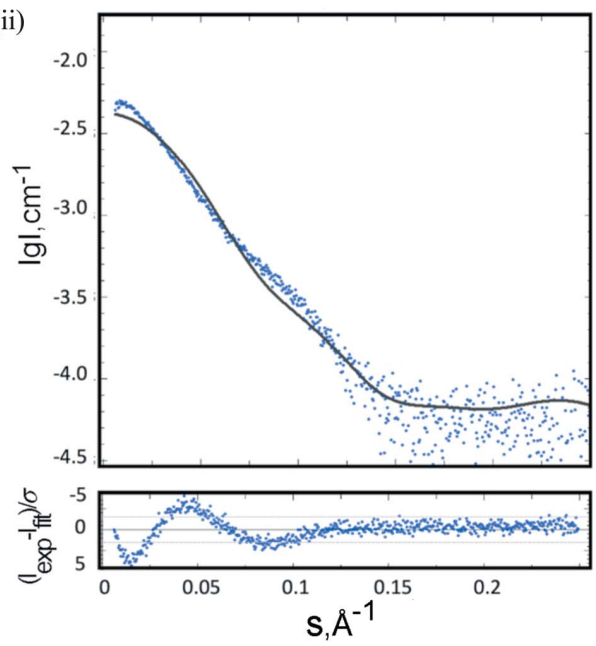

(iv)

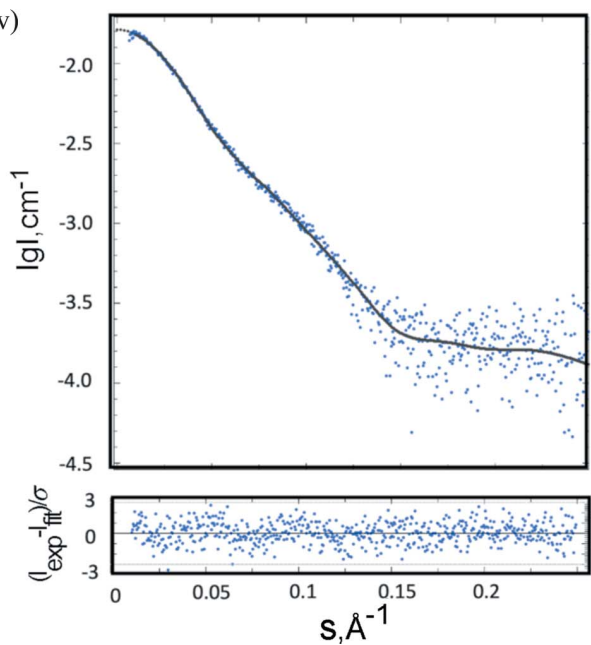

Figure 3

MEMPROT and DADIMODO modelling results. (a) Model and scattering fit for the EptA:DDM complex and (b) the EptA:DPC complex. (a) (i) Representative MEMPROT generated model for the EptA:DDM complex. The atomic structure of EptA is represented in cartoon mode and coloured grey. The DDM corona around EptA is represented as pale blue spheres. (a) (ii) Comparison of the SAXS experimental result (red dots) with the MEMPROT calculated scattering curve (black solid line). The lower inset shows the error-weighted residual difference plot for the experimental SAXS model and the MEMPROT generated model (red dots). (b) (i) Representative MEMPROT generated model for the EptA:DPC complex. The atomic structure of EptA is represented in cartoon mode and coloured grey. The DPC corona around EptA is represented as orange spheres. (b) (ii) Comparison of the SAXS experimental result (blue dots) with the MEMPROT calculated scattering curve (black solid line). The lower inset shows the error-weighted residual difference plot for the experimental SAXS data and the MEMPROT generated model (blue dots). (b) (iii) Model of the EptA:DPC complex obtained with $D A D I M O D O$, assigning the soluble domain and transmembrane domain as rigid bodies connected by a flexible bridging helix and linker region. The atomic structure of EptA is represented in cartoon mode and coloured grey and the DPC corona around EptA is represented as orange spheres. (b) (iv) The lower inset shows the error-weighted residual difference plot for the experimental SAXS data and the $D A D I M O D O$ generated model (blue dots). 
Though the DPC data were very well described by single models calculated by the hybrid procedure, systematic deviations were observed at higher angles for the data of the protein in DDM micelles [Fig. 4(a) and 4(b) panel (ii)]. This

(a) (i)

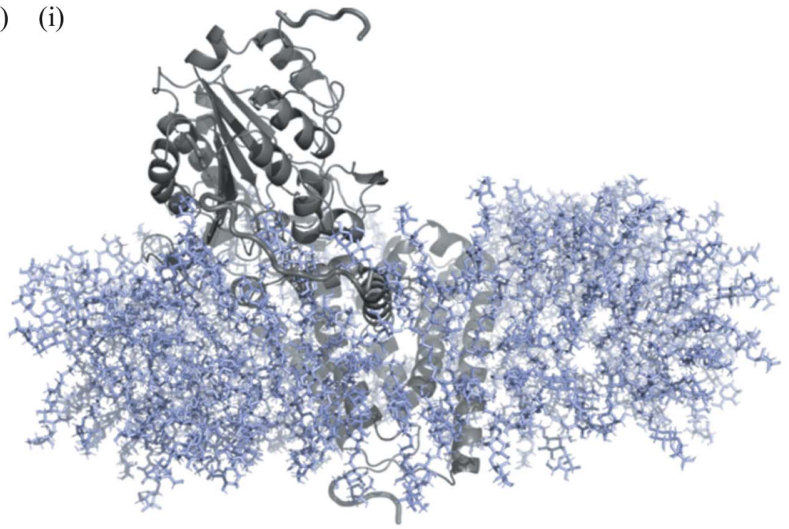

(ii)
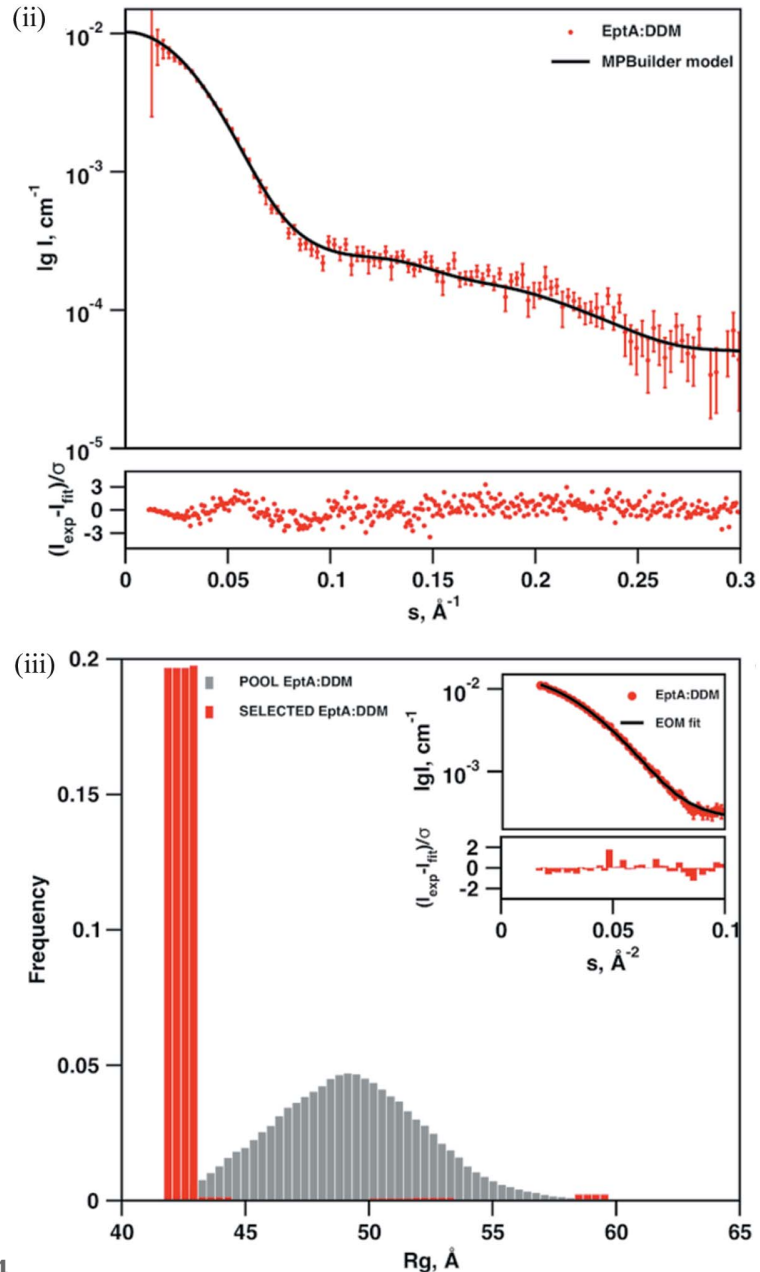

Figure 4 led us to speculate that intrinsic flexibility of EptA may differ between the two detergent conditions and that a structural ensemble may better represent the conformation of the protein in solution. EOM (Bernadó et al., 2007) was applied to
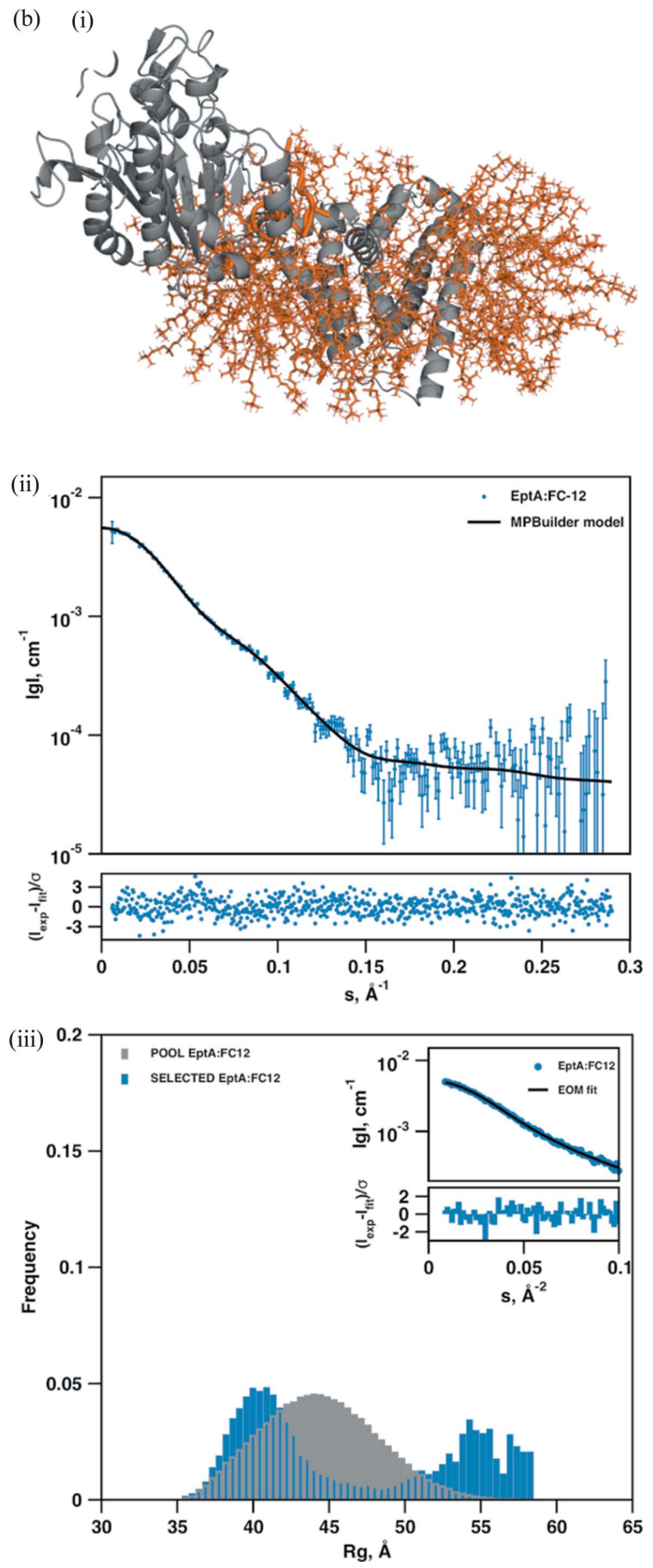

Hybrid SAXS modelling results and flexibility analysis. Results for the (a) EptA:DDM complex and (b) EptA:DPC complex. (i) Hybrid SAXS models generated for each of the complexes using the combined MPBuilder/PACKMOL/CORAL approach. The atomic structure of EptA is represented in cartoon mode and coloured grey. The detergent corona around EptA is represented as pale blue and orange coloured lines for DDM and DPC, respectively. (ii) Comparison of the SAXS experimental data (coloured red and blue for EptA:DDM and EptA:DPC, respectively) with the scattering curves computed from the best fitting hybrid models (black solid line). The lower insets show the error-weighted residual difference plot for the fits. (iii) Flexibility analysis of the complexes using the EOM. Distance profiles $\left(R_{\mathrm{g}}\right)$ of the random pools of PDC conformations generated (grey histograms) and of the selected ensembles of conformations that best fit the SAXS data (red and blue histograms for EptA:DDM and EptA:DPC, respectively). The fits of the selected ensembles to the experimental data and residuals are shown in the inset. For EptA:DDM the selected ensemble consists of a narrow population of similar compact conformations, and two wide populations of both compact and extended conformations are selected for EptA:DPC, indicating significant conformational flexibility. 
investigate the flexibility of EptA in DDM and DPC micelles. $E O M$ constructs a pool of randomized conformations, using high-resolution structure components as rigid bodies with linkers and terminal regions modelled as flexible dummy residues. From this pool ensembles of conformers are selected through a series of elitism steps, with the final ensemble providing a computed scattering pattern that best fits the experimental data. For both EptA detergent complexes the bridging linker between the soluble and transmembrane domains (including the detergent corona as modelled in the $M P B u i l d e r / P A C K M O L$ protocol) was defined as flexible and a pool of randomized conformations were generated. The selection protocol identified ensembles that best fit the scattering data and the distance distributions of these ensembles compared with that of the initial pool [Fig. 4(a) and 4(b) panel (iii)]. For EptA:DDM the distribution of the selected ensemble is narrow and clearly shifted to small $R_{\mathrm{g}}$ values, indicating that, under these conditions, EptA adopts a small range of compact conformations. The metric of flexibility $R_{\text {flex }}$ is $34.8 \%$, compared with $89.0 \%$ calculated for the pool, indicating that the EptA is not significantly flexible in DDM. In contrast, for EptA:DPC two broad populations of conformations are observed in the distribution at low $R_{\mathrm{g}}$ and high $R_{\mathrm{g}}$ values and the calculated $R_{\text {flex }}$ is $91.6 \%$, compared with $88.6 \%$ for the pool. Thus, in DPC EptA exists in a range of conformations and is significantly more flexible. From this analysis the conformational flexibility of EptA was determined to be significantly larger in DPC than in DDM micelles, with the latter again clearly showing a preference for compact structures.

\subsection{Characterizing the flexible regions of EptA mutants in} detergent micelles by intrinsic fluorescence analysis

Previously, intrinsic tryptophan fluorescence studies were carried out using wild type (WT) EptA, in order to probe conformational states of the enzyme in different detergent micelle environments (Anandan et al., 2017). While this provided information regarding conformational flexibility of the molecule as a whole it did not give information as to the specific localization of movement with respect to individual domains. EptA contains six tryptophan residues: Trp247, Trp320 and Trp484 localized in the soluble domain, Trp126 and Trp148 in the transmembrane domain, and Trp207 in the bridging helix. In order to probe the conformational states more fully, the tryptophan residues localized in the transmembrane domain and the bridging helix were mutated to phenylalanine side chains, leaving only the soluble domain tryptophan residues intact resulting in a triple mutant of the enzyme $\left(\right.$ Ept $\left._{\text {Trp126/148/207Phe }}\right)$. Additionally, the tryptophans localized only in the transmembrane domain were mutated to phenylalanines resulting in a double mutant $\left(\mathrm{EptA}_{\mathrm{Trp} 126 / 148 \mathrm{Phe}}\right)$, leaving the soluble domain tryptophan residues as well as the tryptophan on the bridging helix intact. To ensure that these mutations were not detrimental to enzyme function, the minimum inhibitory concentration (MIC) of each mutant to a model CAMP, polymyxin $\mathrm{B}$, was examined. The $N$. meningitidis
NMB $\Delta$ eptA strain had an MIC of $0.19 \mu \mathrm{g} \mathrm{ml}^{-1}$, and expression of both mutants in this strain could restore polymyxin B resistance to the same level as the WT NMB strain (MIC of $384 \mu \mathrm{g} \mathrm{ml}^{-1}$ ), indicating the two mutants were fully functional (Table S3).

Each of these mutants was recombinantly expressed, solubilized and purified in both DDM and DPC micelles, and analysed by circular dichroism (CD) spectroscopy to ascertain the folded state of the protein relative to that of the wild-type enzyme under identical detergent conditions (Fig. S4). The double and triple mutants maintained the secondary structure profile observed for the WT enzyme in both detergent micelles, suggesting a conservation of the fold and secondary structure despite the mutations.

Iodide, a hydrophilic quencher, was used to probe the tryptophan environment of EptA solubilized and purified in the different detergent micelles. The intrinsic tryptophan fluorescence emission spectra of EptA in a native folded state and denatured in the presence of $4 M$ guanidinium- $\mathrm{HCl}$ and $25 \mathrm{~m} M \beta$-mercaptoethanol are shown in Fig. 5(a) panels (i) and (iii), and 5(b) panels (i) and (ii). As expected, the fluorescence signals from the double and triple mutants were diminished relative to the wild-type enzyme, since they now have four and three tryptophan residues, respectively, whereas the wild-type enzyme has six tryptophan residues.

A comparison of the emission spectra for folded proteins in $0.5 \mathrm{M} \mathrm{KCl}$ provides insights into the tryptophan microenvironment of each domain, independent of iodide quenching. The triple mutant in DDM micelles was the most blue-shifted sample with a $\lambda_{\max }$ of $335 \mathrm{~nm}$, indicating the burial of tryptophan residues within the hydrophobic core of the soluble domain [Fig. 5(a) panel (i)]. Solubilization of enzymes in DPC micelles resulted in a red-shift compared with DDM solubilized protein, with the WT, double mutant and triple mutant shifting 7,8 and $4 \mathrm{~nm}$, respectively [Fig. $5(a)$ panels (i) and (iii)]. This suggests that EptA in DPC micelles adopt a more open structure with greater overall solvent exposure of the tryptophan residues than protein in DDM micelles. Furthermore, the $8 \mathrm{~nm}$ red-shift of the double mutant compared with the $4 \mathrm{~nm}$ red-shift of the triple mutant in the two different detergent micelles suggests a more hydrophilic environment in the region of the bridging helix for the protein in DPC micelles as the presence of the tryptophan residue on the bridging helix is the only difference between these mutants. Further support for the role of the bridging helix in opening the structure is evident when comparing each mutant in DPC micelles; the $\lambda_{\max }$ of the triple mutant was $339 \mathrm{~nm}$ whereas the double mutant, which still contained the tryptophan residue on the bridging helix, was red-shifted by $10 \mathrm{~nm}$ to $349 \mathrm{~nm}$.

In all cases, the denatured samples exhibited a $\lambda_{\max }$ of at least $355 \mathrm{~nm}$, a dramatic red-shift from the native folded protein, demonstrating that the tryptophan side chains are positioned in very hydrophilic environments, consistent with an unfolded protein [Fig. 5(b)]. The $\lambda_{\max }$ of the denatured enzyme was similar in DDM and DPC (355-357 nm for WT EptA, $359 \mathrm{~nm}$ for the double mutant and $358 \mathrm{~nm}$ for the triple 
(a)

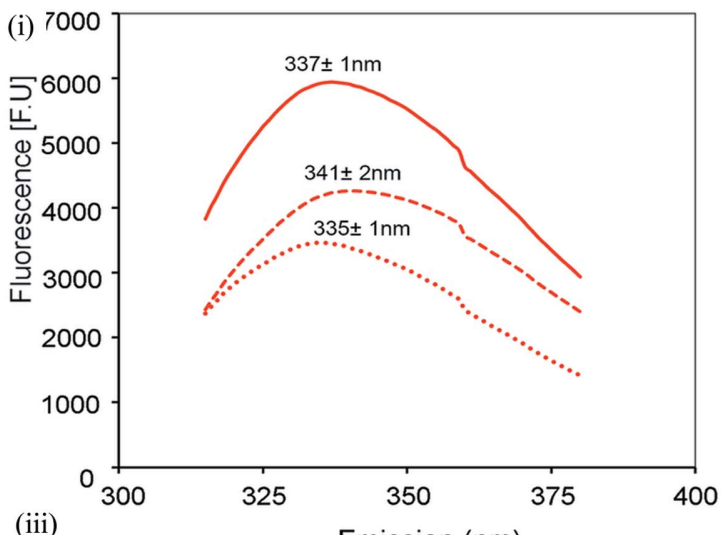

(iii)

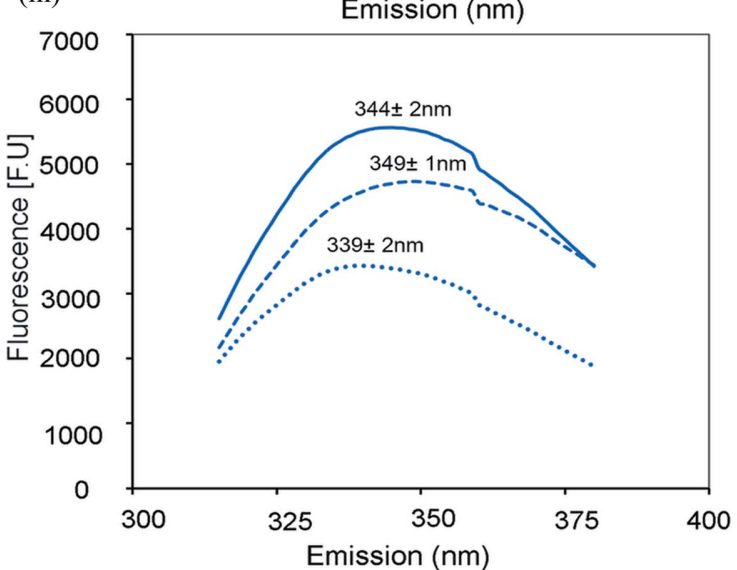

(b) (i) 7000

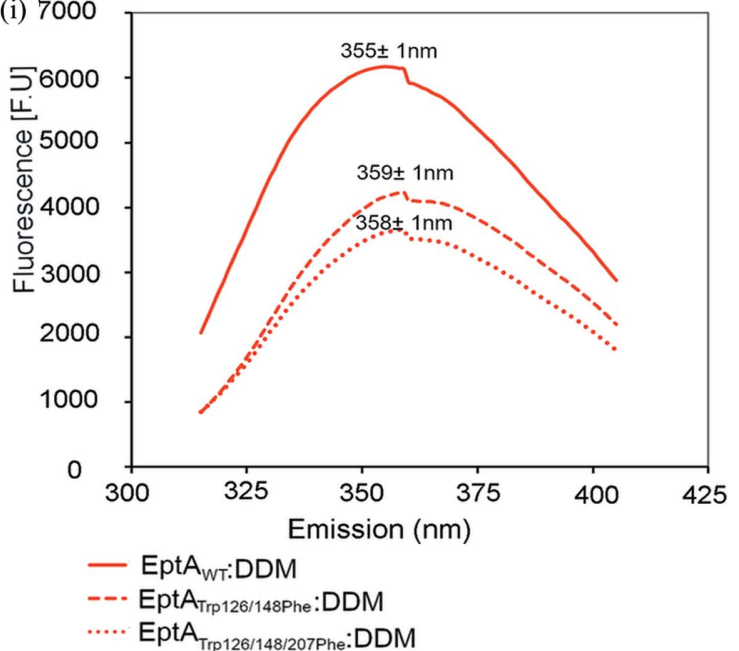

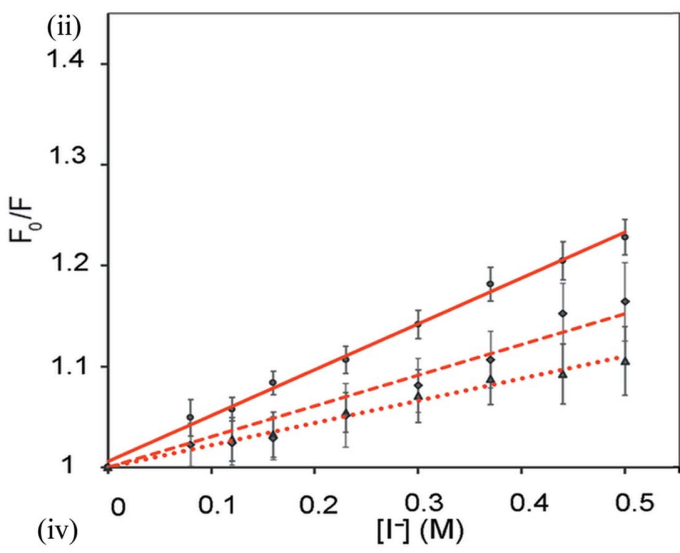
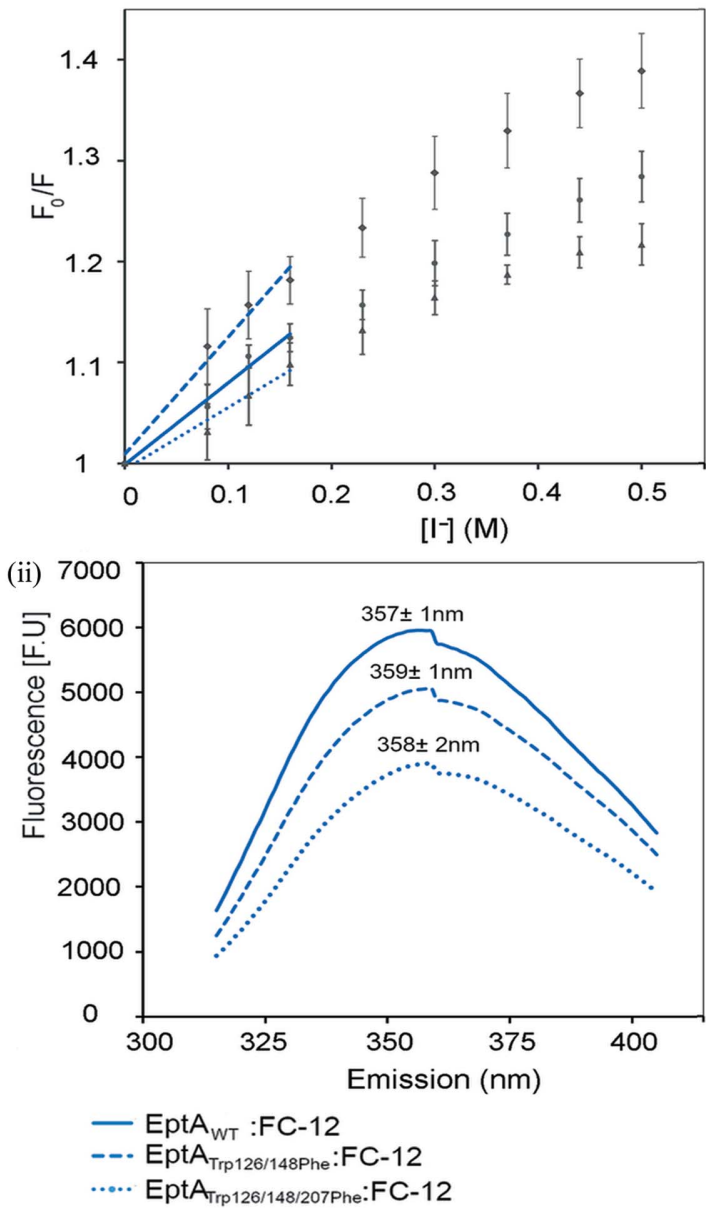

Figure 5

Intrinsic fluorescence spectra and quenching profiles for EptA in different detergent micelles. (a) Fluorescence spectra and Stern-Volmer plots for native folded enzyme and $(b)$ fluorescence spectra for the denatured enzyme. The emission wavelength maxima $\left(\lambda_{\max }\right)$ for each condition are indicated above each curve. The red and blue coloured lines represent enzyme in DDM and DPC micelles, respectively. The solid lines represent Ept $\mathrm{A}_{\mathrm{WT}}$, the

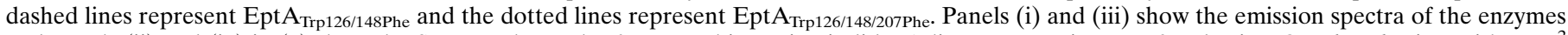
and panels (ii) and (iv) in (a) show the Stern-Volmer plot for quenching using iodide. A linear regression was fitted using GraphPad prism with an $R^{2}$ value above 0.95 . Where a non-linear relationship was observed, only the initial linear slope was fitted.

mutant). This consistency indicates that any detergent-driven conformational differences are absent after denaturation.

Fluorescence-quenching experiments with KI support the results obtained from the emission spectra. Quantitative analysis of the effect of quenching was undertaken by analyzing the Stern-Volmer plots for each protein and deriving the Stern-Volmer constants $\left(K_{\mathrm{SV}}\right)$ [Fig. 5(a) panels (ii) and (iv), Table 2]. Quenching studies were not required for denatured protein, as the emission spectra already demonstrated that these proteins had unfolded to the same extent. The Stern-Volmer plots of the WT and mutant proteins in DDM yielded a linear quenching relationship over the entire range of KI concentrations examined. In these cases, a simple collisional mechanism is used to describe the process of 
Table 2

Summary of the Stern-Volmer constants $\left(K_{\mathrm{SV}}\right)$ obtained from the intrinsic tryptophan fluorescence quenching of EptA and mutants with iodide.

\begin{tabular}{lll}
\hline & \multicolumn{1}{l}{$K_{\mathrm{SV}} \dagger\left(\mathrm{M}^{-1}\right)$} \\
\cline { 2 - 3 } Detergent & $\mathrm{DDM}$ & $\mathrm{DPC}$ \\
\hline EptA $_{\mathrm{WT}}$ & $0.47 \pm 0.02$ & $0.80 \pm 0.11 \neq$ \\
EptA $_{\text {Trp126/148Pe }}$ & $0.30 \pm 0.04$ & $1.23 \pm 0.21 \neq$ \\
EptA $_{\text {Trp126/148/207Phe }}$ & $0.22 \pm 0.01$ & $0.57 \pm 0.14 \ddagger$ \\
\hline
\end{tabular}

$\dagger$ The mean $K_{\mathrm{SV}}$ value is shown with a $95 \%$ confidence interval. $\$$ Where SternVolmer plots exhibited downwards curvature, $K_{\mathrm{Sv}}$ values were calculated from the initial linear slope.

tryptophan quenching by iodide. The Stern-Volmer plots corresponding to the WT and mutant proteins in DPC displayed negative curvature which suggests that EptA in the DPC micelle exhibits considerable mobility such that the tryptophan residues often sit at the micelle surface (Hill et al., 1986). The $K_{\mathrm{SV}}$ values of folded WT EptA were larger than both mutants in DDM micelles and the triple mutant in DPC micelles [Fig. 5(a) panels (ii) and (iv), Table 2]. Additionally, $K_{\mathrm{SV}}$ was larger for WT EptA and for each of the mutants in DPC micelles than those in DDM micelles, suggesting an overall increase in hydrophilicity and quencher accessibility of the tryptophan residues for the protein in DPC micelles. Unlike EptA in DDM micelles, the double mutant exhibited the highest $K_{\mathrm{SV}}$ value for protein in DPC micelles, demonstrating an increase in solvent exposure of the bridging helix. The location of Trp207 on the bridging helix and the structural conformation of the protein in DPC micelles provides more accessibility of this tryptophan to the KI quencher than the transmembrane domain tryptophan residues (Trp126 and Trp149). Indeed, WT EptA that retained the transmembrane domain tryptophan residues exhibited a lower $\mathrm{K}_{\mathrm{SV}}$ value in DPC.

\section{Discussion}

The enzyme chemistry required for lipid A modification by EptA involves the binding of two lipid substrates: phosphatidylethanolamine as a donor lipid and lipid A as a receiver lipid for the PEA moiety. The enzyme first transfers the PEA moiety from the donor lipid to an active-site threonine (Thr280) to make a PEA-bound enzyme intermediate with the release of diacylglycerol. Lipid A must then bind to the enzyme to enable the transfer of PEA from the enzyme intermediate to make the final modified lipid A product. Previously, our crystallographic structure of EptA, where crystals were grown from recombinant enzyme purified in DDM micelles, showed a closed structure that was able to accommodate a DDM molecule in the binding site (Anandan et al., 2017). The O3B of this DDM molecule is positioned identically to that of the phosphate oxygen atom covalently bonded to the side chain of the active-site Thr280 in the truncated soluble domain structure of EptA (Wanty et al., 2013). Further examination of the structure reveals that an enzyme-linked PEA molecule can be accommodated between
Thr280 and Glu114, located in the membrane domain. Indeed, this glutamate side chain is well positioned to form a hydrogen bond with the amine of PEA (Anandan et al., 2017). Based on these observations we hypothesized that the bound DDM molecule mimicked the phosphatidylethanolamine substratebound conformation of the enzyme. However, this conformation would not accommodate the binding of lipid A near to the PEA-bound Thr280 so we presumed that the protein must be able to adopt different conformational states in solution to enable PEA transfer. Molecular dynamic simulations of EptA embedded in the PE/PG system showed that the overall conformation of the enzyme remained close to the starting structure in four of the six simulations, though in two simulations the soluble domain dissociated from the membrane domain and rolled over the membrane surface interacting with the lipid polar heads (Anandan et al., 2017). This suggested that the protein is capable of adopting different conformations and the current study was undertaken to interrogate the flexibility of EptA by SEC-SAXS and intrinsic fluorescence solution studies. WT EptA and mutant forms of the enzyme, where selective tryptophan residues were replaced with phenylalanines, were solubilized and purified in two different detergent systems, namely DDM and DPC, separately.

DDM and DPC detergents contain a 12-carbon acyl chain and a maltoside and phosphocholine head group, respectively. The structural integrity of EptA in these detergent micelles was validated by $\mathrm{CD}$ analyses, differential scanning fluorimetry, and limited proteolysis with trypsin and chymotrypsin (Anandan et al., 2017). The CD spectra of WT protein in both DDM and DPC micelles show a similar profile, suggesting that the secondary structure of EptA is conserved in both detergent micelles. Furthermore, both EptA:DDM and EptA:DPC exhibit distinct melting temperatures suggesting that the enzyme retains a stable tertiary structure in these two detergent systems (Anandan et al., 2017). EptA:DDM and EptA:DPC resisted both trypsin and chymotrypsin digestion and showed distinct bands up to $12 \mathrm{~h}$ further supporting that the protein remains folded in the two detergent systems.

Given that EptA maintains its structural integrity in the detergent micelles used for this study, the results presented here using two different biophysical methods aimed at probing flexibility of the enzyme provide further experimental evidence suggesting that the enzyme is structurally dynamic. For these studies MALS analysis was first performed in order to assess whether the samples were amenable to SAXS studies. However, careful analysis indicated a discrepancy in the molecular weight and thus the MALS data were used mostly to confirm a rough stoichiometry of one protein molecule per micelle, but were not sufficient to confirm the ratio for detergent to protein, most likely due to changes in the concentration between the MALS analysis and the SECSAXS analysis. All modelling-based analyses used the molecular weight calculation obtained directly from the SECSAXS experiment. The Guinier plot from the SEC-SAXS data showed no evidence of aggregation or unfolding which would have been apparent at low $s$. An increase in flexibility of EPtA in DPC was apparent from the Kratky plots. Further 
modelling using multiple algorithms indicated that the models obtained were consistent with folded protein, based on the core of the crystal structure, and these models recapitulate the SAXS data closely. Intriguingly, the analyses of the SAXS data using various algorithms have converged to similar models which match well with the results obtained from the MD simulations of EptA in a membrane environment (Anandan et al., 2017).

Using different tryptophan mutations, the source of the conformational flexibility can be localized to the bridging helix, connecting the soluble domain to the transmembrane domain of the structure. The $K_{\mathrm{SV}}$ constant obtained from the Stern-Volmer plots reflects the accessibility of the quencher to the tryptophan fluorophore and will be smaller with steric hindrance of quencher-fluorophore collisions, if the tryptophan residue is located in a buried crevice or within the hydrophobic protein core (Lakowicz, 2006). The larger conformational flexibility of EptA in DPC micelles as seen from the Stern-Volmer analysis is consistent with the models determined by EOM analysis. In general, the analysis supports the structural observations that the tryptophan residues in the soluble domain are more buried than in the transmembrane domain for protein in each detergent environment since the transmembrane domain tryptophan residues are only present in the WT enzyme. Additionally, the results provide evidence that the conformational flexibility of EptA is localized at the bridging helix and that this flexibility enables the two domains to roll away from one another while the helix becomes more solvent exposed.

A caveat of these studies is that DPC is notoriously well known for causing destabilizing effects in other membrane systems, and thus these results will need further investigation to confirm the exact physiological relevance of the open and closed conformations that we have observed. Nevertheless, the results do provide some insights into the flexible nature of the protein and, intriguingly, align with the results of previous in silico experiments that suggest a flexible domain structure (Anandan et al., 2017). This offers some insights into how EptA might adopt conformational poses that are conducive to binding the different substrates, phosphatidylethanolamine and lipid A, and enabling the transfer chemistry to occur.

We propose these movements are an essential feature needed for the protein to perform its unique chemistry. The bridging helix acts like a hinge region in the structure that enables the protein to open up, exposing the soluble domain and the $\mathrm{Zn}^{2+}$ ion binding site near to the location of the PEAbound Thr280 residue of the enzyme intermediate. This more open structure would provide an enzyme state that allows the larger sized lipid A substrate to access the PEA bound enzyme intermediate.

Thus, by using two different solution-based studies in conjunction with site-directed mutagenesis, a detailed molecular picture of EptA is emerging which helps us to better understand the enzyme chemistry and will be valuable in future drug design strategies for effective inhibitors of the enzyme as novel therapeutic agents to treat MDR bacterial infections. Additionally, the combination of SEC-SAXS and $a b$ initio and rigid-body modelling methods described in this study provides a methodology-based framework for interrogating solution structures of other membrane protein systems solubilized in detergents or nanodiscs.

\section{Acknowledgements}

We also thank Dr Shakeel Mowlaboccus for assistance in the design of the Trp mutant DNA constructs as well as Dr Susannah Piek and Ms Emily Kibble for the design of the NMB $\triangle$ eptA strain and the shuttle vectors required for the polymyxin sensitivity assays.

\section{Funding information}

This work was supported by grant funding from the National Health and Medical Research Council of Australia (grant Nos. APP1003697; APP1078642 awarded to AV and CMK). We acknowledge the Australian Research Council for Infrastructure grant support (LE120100092 awarded to AV). We thank the Australian Synchrotron for access to the SAXS/ WAXS beamline.

\section{References}

Anandan, A., Evans, G. L., Condic Jurkic, K., O’Mara, M. L., John, C. M., Phillips, N. J., Jarvis, G. A., Wills, S. S., Stubbs, K. A., Moraes, I., Kahler, C. M. \& Vrielink, A. (2017). Proc. Natl Acad. Sci. USA, 114, 2218-2223.

Anaya-López, J. L., López-Meza, J. E. \& Ochoa-Zarzosa, A. (2013). Crit. Rev. Microbiol. 39, 180-195.

Bernadó, P., Mylonas, E., Petoukhov, M. V., Blackledge, M. \& Svergun, D. I. (2007). J. Am. Chem. Soc. 129, 5656-5664.

Chipot, C., Dehez, F., Schnell, J. R., Zitzmann, N., Pebay-Peyroula, E., Catoire, L. J., Miroux, B., Kunji, E. R. S., Veglia, G., Cross, T. A. \& Schanda, P. (2018). Chem. Rev. 118, 3559-3607.

Cox, A. D., Wright, J. C., Li, J., Hood, D. W., Moxon, E. R. \& Richards, J. C. (2003). J. Bacteriol. 185, 3270-3277.

Dias Mirandela, G., Tamburrino, G., Ivanović, M. T., Strnad, F. M., Byron, O., Rasmussen, T., Hoskisson, P. A., Hub, J. S., Zachariae, U., Gabel, F. \& Javelle, A. (2018). J. Phys. Chem. Lett. 9, 3910-3914.

Durand, D., Vivès, C., Cannella, D., Pérez, J., Pebay-Peyroula, E., Vachette, P. \& Fieschi, F. (2010). J. Struct. Biol. 169, 45-53.

Evrard, G., Mareuil, F., Bontems, F., Sizun, C. \& Perez, J. (2011). J. Appl. Cryst. 44, 1264-1271.

Franke, D., Petoukhov, M. V., Konarev, P. V., Panjkovich, A., Tuukkanen, A., Mertens, H. D. T., Kikhney, A. G., Hajizadeh, N. R., Franklin, J. M., Jeffries, C. M. \& Svergun, D. I. (2017). J. Appl. Cryst. 50, 1212-1225.

Gasteiger, E., Hoogland, C., Gattiker, A., Wilkins, M., Appel, R. \& Bairoch, A. (2005). The Proteomics Protocols Handbook, pp. 571607. Totowa, NJ: Humana Press.

Gimpl, K., Klement, J. \& Keller, S. (2016). Biol. Proced. Online, 18, 4.

Hajizadeh, N. R., Franke, D., Jeffries, C. M. \& Svergun, D. I. (2018). Sci. Rep. 8, 7204.

Hill, B. C., Horowitz, P. M. \& Robinson, N. C. (1986). Biochemistry, 25, 2287-2292.

Huang, J., Zhu, Y., Han, M. L., Li, M., Song, J., Velkov, T., Li, C. \& Li, J. (2018). Int. J. Antimicrob. Agents, 51, 586-593.

Hwang, P. M., Bishop, R. E. \& Kay, L. E. (2004). Proc. Natl Acad. Sci. USA, 101, 9618-9623.

Imai, S., Osawa, M., Mita, K., Toyonaga, S., Machiyama, A., Ueda, T., Takeuchi, K., Oiki, S. \& Shimada, I. (2012). J. Biol. Chem. 287, 39634-39641.

Janik, A., Juni, E. \& Heym, G. A. (1976). J. Clin. Microbiol. 4, 71-81. 
John, C. M., Liu, M., Phillips, N. J., Yang, Z., Funk, C. R., Zimmerman, L. I., Griffiss, J. M., Stein, D. C. \& Jarvis, G. A. (2012). Infect. Immun. 80, 4014-4026.

Kahler, C. M., Nawrocki, K. L., Anandan, A., Vrielink, A. \& Shafer, W. M. (2018). BMC Genomics, 9, 1922.

Kikhney, A. G. \& Svergun, D. I. (2015). FEBS Lett. 589, 2570-2577.

Kirby, N., Cowieson, N., Hawley, A. M., Mudie, S. T., McGillivray, D. J., Kusel, M., Samardzic Boban, V. \& Ryan, T. M. (2016). Acta Cryst. D72, 1254-1266.

Konarev, P. V., Volkov, V. V., Sokolova, A. V., Koch, M. H. J. \& Svergun, D. I. (2003). J. Appl. Cryst. 36, 1277-1282.

Koutsioubas, A. (2017). Biophys. J. 113, 2373-2382.

Koutsioubas, A., Berthaud, A., Mangenot, S. \& Pérez, J. (2013). J. Phys. Chem. B, 117, 13588-13594.

Lakowicz, J. R. (2006). Principles of Fluorescence Spectroscopy, 3rd ed. Boston, MA: Springer.

Lipfert, J., Columbus, L., Chu, V. B., Lesley, S. A. \& Doniach, S. (2007). J. Phys. Chem. B, 111, 12427-12438.

Lomize, M. A., Pogozheva, I. D., Joo, H., Mosberg, H. I. \& Lomize, A. L. (2012). Nucleic Acids Res. 40, D370-D376.

Martínez, L., Andrade, R., Birgin, E. G. \& Martínez, J. M. (2009). J. Comput. Chem. 30, 2157-2164.

Mertens, H. D. \& Svergun, D. I. (2010). J. Struct. Biol. 172, 128-141.

Molodenskiy, D. S., Svergun, D. I. \& Mertens, H. D. T. (2021). J. Mol. Biol. 433, 166888.

Mylonas, E. \& Svergun, D. I. (2007). J. Appl. Cryst. 40, s245-s249.

Panjkovich, A. \& Svergun, D. I. (2018). Bioinformatics, 34, 19441946.

Pérez, J. \& Koutsioubas, A. (2015). Acta Cryst. D71, 86-93.

Peschel, A. (2002). Trends Microbiol. 10, 179-186.
Petoukhov, M. V., Franke, D., Shkumatov, A. V., Tria, G., Kikhney, A. G., Gajda, M., Gorba, C., Mertens, H. D. T., Konarev, P. V. \& Svergun, D. I. (2012). J. Appl. Cryst. 45, 342-350.

Pollock, J. F., Ashton, R. S., Rode, N. A., Schaffer, D. V. \& Healy, K. E. (2012). Bioconjugate Chem. 23, 1794-1801.

Rambo, R. P. \& Tainer, J. A. (2011). Biopolymers, 95, 559-571.

Receveur-Brechot, V. \& Durand, D. (2012). Curr. Protein Pept. Sci, 13, 55-75.

Ryan, T. M., Trewhella, J., Murphy, J. M., Keown, J. R., Casey, L., Pearce, F. G., Goldstone, D. C., Chen, K., Luo, Z., Kobe, B., McDevitt, C. A., Watkin, S. A., Hawley, A. M., Mudie, S. T., Samardzic Boban, V. \& Kirby, N. (2018). J. Appl. Cryst. 51, 97-111.

Sengottaiyan, P., Petrlova, J., Lagerstedt, J. O., Ruiz-Pavon, L., Budamagunta, M. S., Voss, J. C. \& Persson, B. L. (2013). Biochem. Biophys. Res. Commun. 436, 551-556.

Svergun, D., Barberato, C. \& Koch, M. H. J. (1995). J. Appl. Cryst. 28 , 768-773.

Svergun, D. I. (1992). J. Appl. Cryst. 25, 495-503.

Takeshita, S., Sato, M., Toba, M., Masahashi, W. \& Hashimoto-Gotoh, T. (1987). Gene, 61, 63-74.

Tria, G., Mertens, H. D. T., Kachala, M. \& Svergun, D. I. (2015). IUCrJ, 2, 207-217.

Trombley, M. P., Post, D. M., Rinker, S. D., Reinders, L. M., Fortney, K. R., Zwickl, B. W., Janowicz, D. M., Baye, F. M., Katz, B. P., Spinola, S. M. \& Bauer, M. E. (2015). PLoS One, 10, e0124373.

Tzeng, Y. L., Datta, A., Kolli, V. K., Carlson, R. W. \& Stephens, D. S. (2002). J. Bacteriol. 184, 2379-2388.

Wanty, C., Anandan, A., Piek, S., Walshe, J., Ganguly, J., Carlson, R. W., Stubbs, K. A., Kahler, C. M. \& Vrielink, A. (2013). J. Mol. Biol. 425, 3389-3402.

Whitten, A. E., Cai, S. \& Trewhella, J. (2008). J. Appl. Cryst. 41, 222226. 